\title{
Emotional Intelligence as an Influence on Ethical Behavior: A Preliminary Study
}

\author{
Francis L. Jeffries \\ And \\ Yonggang Lu \\ University of Alaska, Anchorage
}

\begin{abstract}
Author Note's
Francis L. Jeffries, College of Business and Public Policy, Business Administration Department, University of Alaska, Anchorage Yonggang Lu, College of Business and Public Policy, Business Administration Department, University of Alaska, Anchorage

This research was made possible by the generous support from the Department of Management and Marketing in the College of Business and Public Policy at the University of Alaska Anchorage. Dr. Jonathan Alevy and the staff at the University of Alaska Anchorage Experimental Economics Laboratory provided lab support for conduct of the study. The authors are grateful for their advice and support.

Correspondence concerning this article should be directed to: University of Alaska, Anchorage, College of Business and Public Policy, Business Administration Department, 3211 Providence Drive Anchorage, AK 99508-8244, Tel: (907) 7864162, Fax: (907) 786-4115, Email: afflj@uaa.alaska.edu
\end{abstract}

\begin{abstract}
Previous research on ethical behavior has used the Defining Issues Test (DIT) (Rest, 1993) measure of cognitive moral development to predict ethical behavior. The DIT measures rational, as opposed to affective, content of the process leading to ethical behavioral choices. This research introduces Emotional Intelligence (EI) in an attempt to explain more variance in ethical behavior. Data is collected in an experimental economics lab where there are cash payoffs for decisions made. Results indicate that EI does explain some variance in ethical behavior.
\end{abstract}




\section{Introduction}

Recent research has called for an empirical examination of the link between Emotional Intelligence (EI) and ethical behavior (Bay \& McKeage, 2006; Mulki, Jaramillo, \& Locker, 2009). There have been some research efforts addressing whether there is a link between EI and ethical behavior for example, Deshpande and Joseph (2009) and Angelidis and Ibrahim (2011). In Deshpande and Joseph's (2009) paper it was reported that there was a significant relationship between EI and ethical behavior of self. Angelidis and Ibrahim (2011) found a correlation between EI and ethical mindset in that those subjects with high EI held beliefs that actions should not be deceptive or harm others while those with low EI were willing to take action that could have negative consequences for others.

These efforts are commendable and advance our understanding of the EI-ethical behavior link. However, the research to date has relied on self-report measures of intent as opposed to observing actual behaviors. Two of the limitations of using self-report as a methodology are that it is subject to self-selection bias and socially desirable responses. In an attempt to avoid these issues we conducted an experiment testing the link between EI and ethical behavior in an experimental economics laboratory where the subjects make decisions and experience real economic consequences for their decisions. This should more accurately simulate an actual situation.

Much previous research on ethics has focused on the moral maturity of individuals using the Defining Issues Test (DIT) (Rest, 1993) to predict ethical behavior. The DIT is a measure of the cognitive process leading to ethical behavioral choices so it is more a measure of the rational, as opposed to the affective or emotional, content of the process. Consistent with the call above for research regarding the link between EI and ethical behavior, other researchers have noted the potential value of including affective content into our explanations of how we make ethical behavioral choices (Gaudine \& Thorne, 2001; Maclagan, 1990; Weaver \& Trevino, 1999).

In this study we develop a new model for understanding the respective influences of Moral Maturity and Emotional Intelligence (EI) on ethical behavioral choices and conduct a test of the theory using a simple decision task, a dictator game, in which one player decides how to allocate funds between themselves and one other person (see Camerer, 2003; Engel 2011 for reviews). The data is collected in an experimental economics lab where there are real cash outcomes for the choices that the participants make. We selected this methodology to increase the significance and consequences of the participant's choices over that of survey research.

The remainder of the paper will proceed as follows. A brief review of the DIT and EI literature and the development of a model for behavioral predictions is presented. Next, the model is tested in an experimental economics lab and the results are presented and discussed. We close with suggested future research directions and implications for management. 


\section{Moral Maturity}

Kohlberg (1969) developed a six stage model of cognitive moral development that is still widely used today. Stage 1 is the stage of punishment and obedience. In this stage right is literal obedience to the letter of the rules and authority. There is no association of right with any higher order or philosophy, but rather with the person who has power. Stage 2 is the stage of individual instrumental purpose and exchange, and is where that which serves one's own needs defines right. Rules and authority take a back seat to what is fair to the individual (in his/her own estimation) at this stage. Decisions are made on the basis of a negotiated outcome that benefits the individual and may or may not conform to the guidelines of the authorities. Stage 3 is the stage of mutual interpersonal expectations, relationships, and conforming where the individual begins to emphasize others rather than him/herself. The primary difference between this stage and the preceding one is the consideration of others in making the decision. The stage of social system and conscience maintenance, stage 4 , is where the individual considers his/her responsibility to society not just other specific people. The stage of prior rights and social contract or utility, stage 5, is where decisions are made based on concern for upholding some basic rights, values, and legal contract with society. The final stage concerns universal ethical principles. The laws, rights, and social agreements gain validity not because of a society's law or custom, but rather because they rest on universal principles. At this stage one's ethical practice does not rely on the organization or society for ethical direction.

Building on Kohlberg's (1969) work, Rest (1993) developed the Defining Issues Test (DIT). The DIT when administered yields a P score that is representative of the respondent's moral reasoning ability (Rest, 1993). Higher P scores on the DIT relate to greater cognitive reasoning ability regarding ethically challenging situations. Actions of those with higher $\mathrm{P}$ scores have been associated with more ethical behavior in numerous situations. For example, in stock trading simulations with insider information those with higher P scores were less likely to use the information to their advantage (Abdolmohammadi \& Sultan, 2002). The DIT has been used extensively in the area of ethics research in business with more than 50 studies published in the Journal of Business Ethics from 1996 to 2005 (Herrington \& Weaven, 2008).

Predicting ethical behavior based primarily on cognitive moral development as measured by the DIT may be limiting our ability to account for variance in ethical behavior and inclusion of an affective component may increase our understanding of what other constructs influence ethical behavior. It has been shown that EI has explanatory power in a variety of situations where behavioral choices are made. For instance, inclusion of EI increased the explained variance in employee performance over that explained by cognitive mental ability alone. Van Rooy and Viswesvaran (2004), in a recent meta-analysis of EI research, found that the most successful employees were likely to have a high level of both general mental ability and emotional intelligence. They concluded that both general mental ability and EI have an impact on behavioral choices leading to performance. Applying this line of reasoning to ethical behavioral choices, the inclusion of EI representing the affective side of making choices has the potential to increase our understanding of ethical behavioral choices and may increase our ability to more accurately predict behavior. 


\section{Emotional Intelligence}

EI is 'the ability to monitor one's own and others' feelings and emotions, to discriminate among them and to use this information to guide one's thinking and actions" (Salovey \& Mayer, 1990: 189). EI is distinct from other forms of intelligence, general intelligence for instance, since it is involved specifically with the management of emotions and emotional content (Jordan, Ashkanasy, Hartel, \& Hooper, 2002; Mayer \& Salovey, 1997). The focus on recognizing and managing emotion makes EI distinct from impression management (Lennox \& Wolfe, 1984; Snyder, 1979) as well.

Several models of emotional intelligence have been developed. One of the most widely accepted was developed by Mayer and Salovey (1997), a four component model of EI. Emotional perception is the ability to be self-aware of emotions and to be able to express emotions and emotional needs accurately to others. Emotional assimilation is the ability of one to distinguish among the different emotions he/she may be feeling and to prioritize those influencing current thought processes. This facilitates one's focus on important information and understanding why the feelings are being experienced. Emotional understanding is the ability to understand complex emotions such as simultaneous feelings of loyalty and betrayal. Emotion management is the regulation of one's emotions. This involves the ability to connect with or to disconnect from a given emotion depending on its appropriateness for a given situation.

In sum, EI is the ability to be aware of one's own emotional state, to regulate those emotions, and to respond to the situation that is the catalyst of the emotion in a way that facilitates acquisition of outcomes that are sought by the individual. The value of EI in organizational life is actively being researched and has shown to be related positively to both personal and organizational outcomes (e.g. Abraham 2000; Jordan et al. 2002; Lam \& Kirby 2002; see also Van Rooy \& Viswesvaran 2004 for a meta-analysis).

\section{EI, Moral Maturity, and Ethical Behavior}

We predict that more ethical behavior will result when a person has higher scores for both moral maturity (P score) and EI (see figure). If one scored higher on the DIT they would be prone to behave in a more ethical manner assuming that they used a purely cognitive process for making the decision. However, as noted by Ajzen (1985), there are both cognitive and affective components to behavioral decisions. If the person was also high in EI then they would be more likely to be attentive to the consequences of the act they are contemplating (Freedman 2002), They would be more likely to do the right thing because it is the right thing to do (Freedman, 2002; Goleman, 1999) acting in concert with their principles and values. The emotional dimension of ethical behavior helps keep the real importance of the issues top of mind ensuring a more likely choice of a more acceptable action (Maclagan, 1990). Thus:

\section{H1: When an individual scores higher on both the DIT and EI it is very likely that they will exhibit more ethical behavior.}




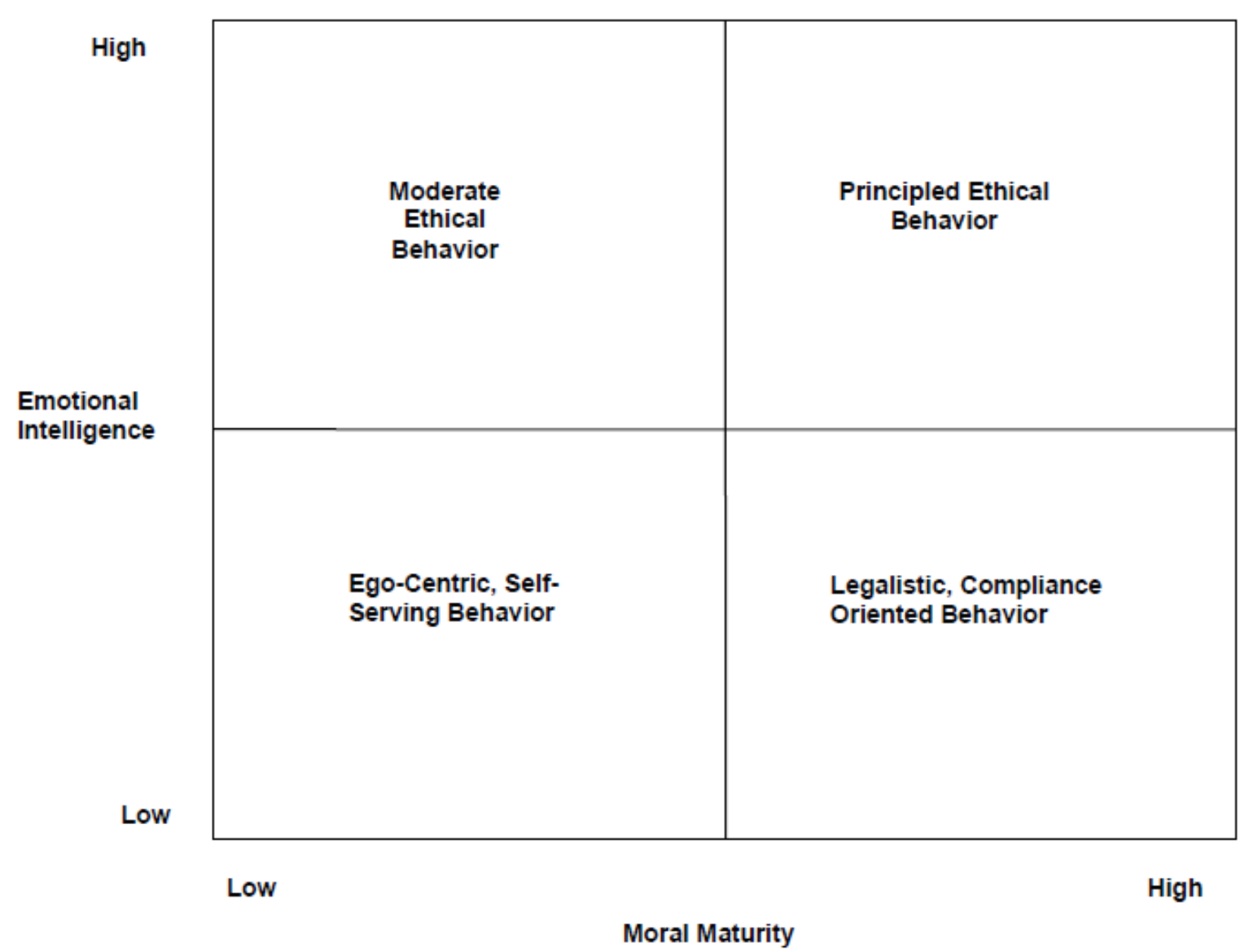

Figure. Combined effects of moral maturity and EI

Conversely, a person who scores lower on both EI and the DIT has the lowest likelihood of ethical behavior. In this case, assuming that the person scored lower on the DIT, concern for others would not be a relevant consideration. If the immediate consequences for the actor were good the act would be justified. Being low on EI as well, considering the long term consequences would not be an issue and considering how the act would impact others and having empathy for them would also be less likely.

The context would be more relevant in these situations as well, since persons who scored low on EI and moral maturity did not consider long term consequences for others and their behavior was legalistic (Ross \& Robertson, 2003). Thus the best one could reasonably expect is compliance with the rules if one was reasonably certain that the act would be observed. If potential negative consequences were avoidable and/or enforcement of the rules was not very certain self-serving behavior will be more likely. In situations where lower moral maturity and EI are present it is expected that the person will be more influenced by the situation and serving their own needs without regard for others. Thus:

H2: When an individual scores low on both the DIT and EI it is very likely that they will exhibit unethical behavior that is ego-centric and self-serving. 
A person who scores low on EI and high on Moral Maturity will be biased toward a cognitive approach to ethical decision making. While there is a chance that the person will actually engage in ethical decision making it has been shown that situational variables may have a relatively large influence (Ross \& Robertson, 2003). For example, a compliance oriented context could result in a self-interested response (Weaver \& Trevino, 1999). While a high score on the DIT indicates an ability to operate at higher levels of moral reasoning, it by no means guarantees it.

Low EI will not strongly support the choice of behaving ethically, so it is likely that there will be lower affective drive to act ethically and even though the person has a relatively high $\mathrm{P}$ score it is likely that they will be more susceptible to situational influences. When taken together these influences will combine to dampen the effect of the cognitive moral reasoning ability of the individual, thus:

H3: When an individual scores high on the DIT and low on EI it is very likely that they will exhibit legalistic compliance oriented behavior resulting in a moderate level of ethical behavior.

If a person scores high on EI and low on the DIT they will use a less advanced cognitive approach to moral reasoning. It is more likely that they will be concerned with what others may think of their decision and at that same time also focus more on the impact the decision will have on themselves.

Taken together the combination will result in a less sophisticated cognitive approach to an ethical behavioral choice that is tempered by an awareness of how the choice will impact others as well as themselves. Thus:

H4: When an individual scores low on the DIT and high on EI it is very likely that they will exhibit behavior resulting in a moderate level of ethical behavior.

\section{Methodology \& Experiment Design}

\section{DIT and EI Scales}

To measure the cognitive ability to make ethical decisions the DIT 2 was used (Rest \& Narvaez, 1998). It was selected because it is a shorter version of the original DIT and has been shown to be comparable in performance. Reliability of the DIT has been consistently in the high .70s to high .80's (Dellaportas, 2006).

Emotional intelligence was measured using Wong and Law's Wong and Law Emotional Intelligence Scale (WLEIS) (Wong \& Law, 2002). The scale is a four-dimensional scale that adheres to Mayer and Salovey's (1997) ability model. The scale has been shown to be reliable $(\alpha=.88)$ (Whitman, Van Rooy, Viswesvaran, \& Kraus, 2009). It has been evaluated by researchers and found to have suitable psychometric properties for EI research (Joseph \& Newman, 2010; Ng, Wang, \& Zalaquett, 2007).

\section{Experiment Design}


We conduct two-player dictator games using a $2 \times 2$ factorial design in which both the choice set and the observability of the dictators' choices are varied, between subjects.

Variation in the choice set arises from the implementation of give $(\mathrm{G})$ and take $(\mathrm{T})$ protocols. Provisional endowments of \$20 (\$10) for Player A (Player B) were given to Player A in two envelopes in the form of $\$ 1$ bills (U.S.). Each envelope was labeled with the player roles and a common numeric identifier for the subject pair. In the give frame Player A was instructed that they could transfer to Player B any amount from $\$ 0$ to their entire endowment of \$20 in one dollar increments, by transferring bills from the A envelope to the B envelope. In the take frame the set of possible transfers is expanded to range from $-\$ 10$ to $\$ 20$, allowing transfers from $\mathrm{B}$ to $\mathrm{A}$ of amounts up to and including the entire endowment.

Variation in the observability of Player A's behavior is created by implementing a protocol that preserves anonymity (A), and a public protocol in which each dictator's decision is observed by all others in the experimental lab $(\mathrm{O})$. In both observable and anonymous conditions, each Player A dropped their B envelope into a sealed box individually after allocation decisions were made. In the observable condition, all envelopes were first collected by the experiment monitor. Each Player A was then individually called to the front of the room, where the amount in Player B's envelope was counted by the experimental monitor and made public by entering the dollar value into a spreadsheet projected at the front of the room. All monies were returned to the B envelope and Player A then dropped the envelope into a sealed box before returning to their seat and rejoining the audience. As in List (2007), Player A and Player B "did not have any contact before, during, or after the session." The audience effects we examine are therefore associated with the observation of dictator decisions by the experimental monitor and the other A players. Three distinct monitors were used, all undergraduates experienced with extensive previous experience in the conduct of experiments. We speculate that the absence of B players and faculty monitors in the audience may weaken audience effects.

Experimental sessions were conducted at the University of Alaska Anchorage Experimental Economics Laboratory. The lab infrastructure includes shielded workstations so that players' actions were not observed while tasked with determining the final contents of the envelopes. Recruitment was conducted through the use of a MooreRecruting database, and a total of 228 dictator decisions are observed. Treatments are indicated by the combination of letters associated with each factor: GA, TA, GO, and TO. Sessions were conducted between June, 2012 and April, 2013. DIT and EI surveys were conducted for each participant and a total of 215 completed surveys were obtained. The order of the implementation of the survey and experiment were randomized across sessions.

\section{Results}

For the purposes of this paper we are reporting only the results relevant to the propositions being tested. We reported the results on the importance of observability across the give and take frames in the dictator game, where we found both audience effects and gender effects, in Alevy, Jeffries, and Lu (2013). 
In total, valid survey and experimental data was collected from 215 recruited participants taking role of Player A. Of the 215 participants, 93 participants played the baseline give game in which 44 participants' actions are observed. One hundred and twenty two participants played the take game in which 58 participants' actions are observed. Demographic information on age, sex, and education level is also collected. The participants' age ranges from 18 to 69 with the median of 22. One hundred and nine participants are female and 106 participants are males. Participant's education background is decoded as integer index from 3 to 13 representing education level from high school to doctoral degree. In accordance with our hypotheses, we identify each participant in one of four quadrants using scores on Wong et al.'s 16-point measure of Emotional Intelligence and the P Score measure of Moral Maturity. We attempt to classify the 217 participants into four blocks: High Moral Maturity and High Emotional Intelligence (HMHE), High Moral Maturity and Low Emotional Intelligence (HMLE), Low Moral Maturity and High Emotional Intelligence (LMHE), Low Moral Maturity and Low Emotional Intelligence (LMLE). Although such classification may be done by simply using the group median scores on the two measures (3.875 for the EI measure and 38 for the Moral Maturity measure) as thresholds, we found our score data collection shows large variability on both measures (coefficient of variation of 11.11 for the EI measure and 43.65 for the Moral Maturity measure). Therefore, we decided to classify only those participants with score on EI (Moral Maturity) lower than the first quartile into LE (LM) group, and those participants with score on EI (Moral Maturity) higher than the third quartile into HE (HM) group. That reduces the number of usable records from 217 to 112 . The classified participants are approximately evenly distributed among the four blocks as shown below.

\begin{tabular}{c|cc} 
& HM & LM \\
\hline HE & 23 & 27 \\
LE & 32 & 30
\end{tabular}

An ANCOVA model of the form of equation (1) is fitted with the collected data

$$
E(\text { Money kept })=\beta_{0}+\beta_{1} \text { Age }+\beta_{2} \operatorname{Sex}+\beta_{3} E d u+\beta_{4} M M+\beta_{5} E I+\beta_{6} M M \times E I,
$$

where age, sex and education are confounding variables, MM and EI are two dummy variables indicating participant of High Moral Maturity vs. Low Moral Maturity and High Emotional Intelligence $v s$. Low Emotional Intelligence respectively. It is necessary to include the confounding variables into our model because one's sex, age and education may have an impact on the person's behavior. However, these effects are not our main interest in this study.

Our measure of ethical behavior is the amount of money kept for oneself in the dictator game. Accordingly, our interest lies in comparison of estimated least squared means of amount of money kept by Player A among the four blocks defined above after controlling for the impact of other unwanted extraneous factors (i.e., age, sex, and education level) on the behavior of participants.

We estimated the least squared or marginal means through model (1) separately for the four different experiments: baseline give game with observed actions, baseline give game with unobserved actions, take game with observed actions and take game with unobserved actions. Meanwhile, in Tables 1-5, results are summarized as $90 \%$ confidence intervals of 
difference between least square means of amount of money kept by Player A in each pair of two blocks.

It is seen that the reported least square mean differences between different groups are relatively much larger under take game with unobserved actions than under other scenarios. The analysis results show that different levels of ethical behavior among participants are more notably observed in the experiment in which participants are given the option of taking money from Player B with their actions unobserved. Under such setting of the experiment, participants of high EI and high Moral Maturity display consistently more ethical behavior than all other participants. Thus, our Hypothesis 1 is consistent with empirical data. In particular, at the $90 \%$ significance level our data can support the conclusion that ethical behavior of HMHE group has significantly more ethical behavior than LMHE group. It shows that difference in ethical behavior between people of high Moral Maturity and people of low Moral Maturity can possibly be further enhanced when they all have high EI. On the other hand, our data cannot statistically confirm that HMHE group has significantly more ethical behavior than HMLE and LMLE groups. That is probably because our dataset has relatively low statistical power due to small sample size. Meanwhile, what's curious to us is that our data showed at the $90 \%$ level that LMHE participants have significantly less ethical behavior than both HMLE and LMLE participants. This finding is inconsistent with what we would expect and is the first empirical study we are aware of that suggests that people of low Moral Maturity can behave more unethically when they have high EI.

Table 1

Confidence interval estimates under give game with observed actions

\begin{tabular}{ccccc} 
& & & \multicolumn{2}{c}{ 90\% Confidence Limits for LSMean(B1)- } \\
B1 & B2 & LSMean(B1)-LSMean(B2) & -3.84 & 9.93 \\
HMHE(1) & LMHE(3) & 3.05 & -8.91 & 2.82 \\
HMHE(1) & HMLE(2) & -3.04 & -7.05 & 5.45 \\
HMHE(1) & LMLE(4) & -0.80 & -12.58 & 0.40 \\
LMHE(3) & HMLE(2) & -6.09 & -9.57 & 1.88 \\
LMHE(3) & LMLE(4) & -3.84 & -2.46 & 6.95 \\
HMLE(2) & LMLE(4) & 2.25 & & \\
\hline \hline
\end{tabular}

Table 2

Confidence interval estimates for give game with unobserved actions

\begin{tabular}{ccccc} 
& & & \multicolumn{2}{c}{ 90\% Confidence Limits for LSMean(B1)- } \\
B1 & B2 & LSMean(B1)-LSMean(B2) & -9.05 & 4.12 \\
HMHE(1) & LMHE(3) & -2.46 & -4.65 & 5.74 \\
HMHE(1) & HMLE(2) & 0.54 & -4.89 & 5.99 \\
HMHE(1) & LMLE(4) & 0.55 & -3.54 & 9.55 \\
LMHE(3) & HMLE(2) & 3.01 & -4.08 & 10.10 \\
LMHE(3) & LMLE(4) & 3.01 & -4.87 & 4.87 \\
HMLE(2) & LMLE(4) & 0.0036 &
\end{tabular}


Table 3

Confidence interval estimates under take game with observed actions

\begin{tabular}{ccccc} 
& & & \multicolumn{2}{c}{ 90\% Confidence Limits for LSMean(B1)- } \\
B1 & B2 & LSMean(B1)-LSMean(B2) & -8.86 & 7.32 \\
\hline HMHE(1) & LMHE(3) & 0.77 & -6.79 & 8.48 \\
HMHE(1) & HMLE(2) & 0.85 & -3.32 & 13.59 \\
HMHE(1) & LMLE(4) & 5.14 & -5.03 & 8.25 \\
LMHE(3) & HMLE(2) & 1.61 & -1.30 & 13.11 \\
LMHE(3) & LMLE(4) & 5.91 & -2.80 & 11.39 \\
HMLE(2) & LMLE(4) & 4.29 & &
\end{tabular}

Table 4

Confidence interval estimates under take game with unobserved actions

\begin{tabular}{ccccc} 
& & & \multicolumn{2}{c}{ 90\% Confidence Limits for LSMean(B1)- } \\
B1 & B2 & LSMean(B1)-LSMean(B2) & -17.26 & -5.91 \\
\hline HMHE(1) & LMHE(3) & -11.59 & -7.64 & 3.59 \\
HMHE(1) & HMLE(2) & -2.02 & -9.70 & 0.19 \\
HMHE(1) & LMLE(4) & -4.75 & 4.06 & 15.07 \\
LMHE(3) & HMLE(2) & 9.56 & 1.65 & 12.02 \\
LMHE(3) & LMLE(4) & 6.84 & -8.10 & 2.64 \\
HMLE(2) & LMLE(4) & -2.73 & &
\end{tabular}

It is also of interest to understand difference in behaviors of participants under the condition of observed action $v s$. unobserved action in both give and take games. To serve this purpose, the second ANCOVA model is suggested in the general conceptual form (2) $E($ Money Kept $)=\beta_{0}+\beta_{1}$ Age $+\beta_{2}$ Sex $+\beta_{3} E d u+\beta_{4}$ Observed, (2) where Observed is a dummy variable indicating the experiment settings of observed action $v s$. unobserved action. Similarly, our interest lies in comparison of estimated least squared means of amount of money kept by Player A between groups of observed action and unobserved action in each of the four blocks. The data results are given in Table 5.

Table 5

Confidence interval estimates comparing observed action with unobserved action 90\% Confidence Limits for LSMean(Unobs)-

\begin{tabular}{cccc} 
B & LSMean(Unobs)-LSMean(Obs) & \multicolumn{2}{c}{ LSMean(Obs) } \\
\hline HMHE & -4.17 & -8.13 & -0.22 \\
LMHE & 0.73 & -4.28 & 5.73 \\
HMLE & 0.51 & -3.43 & 4.45 \\
LMLE & 5.65 & 1.38 & 9.93 \\
\hline \hline
\end{tabular}


Overall, the data suggest that the participants of high EI and high Moral Maturity have significantly more ethical behaviors when their actions are not observed. Conversely, the participants of low EI and low Moral Maturity generally display significantly less ethical behaviors when their actions are not observed.

In summary, the unobserved taking condition is of interest for the purpose of reporting results of our hypotheses since it will be the most indicative of what the subjects really desire to do. Hypothesis 1 predicted that those scoring high on both the DIT and EI (HMHE) would perform more ethically than all others. In the unobserved taking condition those who scored high on the DIT and EI took less than all others providing support for the hypothesis to some extent.

Hypothesis 2 predicted that those who scored low on both the DIT and EI (LMLE) would behave less ethically than the others. For this we have a mixed result. They took more than those who scored high on the DIT and EI, but significantly less than those who scored low on the DIT and high on EI. We did not expect this result.

Hypothesis 3 predicted that those who scored high on the DIT and low on EI (HMLE) would display a moderate level of ethical behavior. There is mild support for this prediction in that we observed this group took more than HMHE group, although not statistically significant at the $90 \%$ level. Meanwhile, it is also seen that this group took less than LMLE group. Those results are within our expectations.

Hypothesis 4 predicted that those who scored low on the DIT and high on EI (LMHE) would display a moderate level of ethical behavior. There is lack of support for this prediction. As expected, LMHE took more than HMHE. However, the difference between LMHE and LMLE is not what we expected in that LMHE actually took more than LMLE. Also that LMHE took significantly more than HMLE is unexpected.

\section{Discussion}

The results are mixed, but encouraging. As expected HMHE resulted in the most ethical behavior of all combinations. The large difference between the HMHE and HMLE groups demonstrated that there is reason to believe that EI does have an impact on ethical behavioral choices.

We observed a large difference between the amount that the HMLE group and the LMHE group took relative to the HMHE group, 2.02 and 11.59 respectively. That provides an indication that the presence of EI may help explain some of the variance in ethical behavioral choices.

While we predicted moderate levels of ethical behavior in both of these conditions (HMLE and LMHE) it appears that they are not going to be balanced in the way the respective combinations influence ethical behavioral choices. In fact, LMHE took significantly more than HMLE. Also, LMLE did not appear to make the most unethical choices as we expected. Only HMHE took less than LMLE. It is odd that these results show 
that high EI may actually stimulate unethical behaviors of people of low Moral Maturity. This is inconsistent with previous research and it may be due to context or sample size. Further research will be necessary to determine the validity of this result.

We have discussed some possibilities regarding why the results came out as they did. It could be that the experiment needs to be modified to incorporate other dimensions of ethical behavioral choice making. Taking may not be a suitable analog for an ethical behavior. There may also be another variable involved that can account for the results, though it is unclear what it would be currently.

\section{Limitations}

The research was conducted in a laboratory setting using an ultimatum game to test ethical choice making and as such it may not have been a perfect analog for actual ethical choice making. In addition, some could argue that giving and taking in this context do not have sufficient relevant ethical content to test the model as presented. Finally yet importantly, the small number of usable data records limits statistical power of our data.

\section{Conclusion and Future Directions}

We conducted an experimental study to test the propositions relating EI to ethical behavioral choices and report intriguing results on the influence of emotional intelligence and moral maturity on behavior in dictator games. In spite of limited statistical power, this study has provided sufficient evidence to indicate that the effect of EI on ethical choice making is worthy of future research. Next efforts should consider use of other large-scale experiments that involve behavioral challenges that more directly tap ethical thinking and consideration of consequences.

Assuming that this line of research continues to increase our understanding of EI on ethical choice making and we find that it is indeed a significant factor, the implications for ethics education in college and business are profound. Academe has been unsuccessful in developing a curriculum that has enough persistence after students leave the institution as evidenced by the research on accounting students (Wright, 1995) and pharmacy students (Latif, 2000). If, in fact, EI explains variance in ethical behavior beyond that explained by the P score, then it would lead to the conclusion that aiding students to develop a higher level of EI in the course of their college careers could lead to lasting gains both in ability to make ethical choices and also overall performance in the workplace.

Finally, as a continuous line of research develops in this area and we continue accumulating knowledge, we advocate that data analysis in future studies be conducted using Bayesian approaches such as what proposed in Lu et al. (2016), instead of the frequentist method in this preliminary study. This will allow our understanding of EI on ethical choice making to be updated through a more coherent mechanism by always incorporating results from preceding studies as informative prior knowledge into a new study. 


\section{References}

Abdolmohammadi, M. and Sultan, J. (2002). Ethical reasoning and the use of insider information in stock trading. Journal of Business Ethics. 37(2); 165-173.

Abraham, R. (2000). The role of job control as a moderator of emotional dissonance and emotional intelligence-outcome relationships. The Journal of Psychology, 134(2): 169-184.

Ajzen, I. (1985). From intentions to actions: A theory of planned behavior. In J. Kuhl \& J. Beckmann (Eds.), Action-control: From cognition to behavior. Heidelberg: Springer.

Alevy, J. E., Jeffries, F. L., and Lu. Y. (2013). Gender- and frame-specific audience effects in dictator games. Economics Letters, 122(4): 50-54.

Angelidis, J. and Ibrahim, N. A. (2011). The impact of emotional intelligence on ethical judgment of managers. Journal of Business Ethics, 99:111-119.

Bay, D. and McKeage, K. (2006). Emotional intelligence in undergraduate accounting students: Preliminary assessment. Accounting Education: An International Journal, 15(4): 439-454.

Camerer, C. F. (2003). Behavioral Game Theory: Experiments in Strategic Interaction. Princeton: Princeton University Press.

Dellaportas, S. (2006). Making a difference with a discrete course on accounting ethics. Journal of Business Ethics, 65(4): 391-404.

Deshpande, S. P. and Joseph, J (2009). Impact of emotional intelligence, ethical climate, and behavior of peers on ethical behavior of nurses. Journal of Business Ethics, 85: 403410.

Engel, C. (2011). Dictator games: a meta study, Experimental Economics, 14, 583-610.

Freedman, J. (2002). What makes up EI? www.6seconds.org.

Gaudine, A. and Thorne, L. (2001), "Emotion and ethical decision-making in organizations," Journal of Business Ethics, 31:175-187.

Goleman, D. (1999). What makes a leader? Harvard Business Review, 76(6): 93-102.

Herington, C. and Weaven, S. (2008). Improving consistency for DIT results using cluster analysis. Journal of Business Ethics, 80(3); 499-514.

Jordan, P. J., Ashkanasy, N. M., Härtel, C. E. J., and Hooper, G. (2002). Workgroup emotional intelligence: Scale development and relationship to team process effectiveness and goal focus. Human Resource Management Review, 12(2): 195-215.

Joseph, D. L. and Newman, D. A. (2010). Discriminant Validity of Self-Reported Emotional Intelligence: A Multitrait-Multisource Study. Educational and Psychological Measurement, 70(4): 672-694

Kolhberg, L. (1969). Stage and sequence: The cognitive developmental approach to socialization. In D.A. Goslin (ed.), Handbook of Socialization Theory and Research. (347-480). New York: Rand McNally.

Lam, L. T. and Kirby, S. L. (2002). Is emotional intelligence an advantage? An exploration of the impact of emotional and general intelligence on individual performance. The Journal of Social Psychology, 142(1): 133-143.

Latif, D. A. (2000), Ethical cognition and selection-socialization in retail pharmacy. Journal of Business Ethics 25(4), 343-357.

Lennox, R. D. and Wolfe, R. N. (1984). Revision of the self-monitoring scale. Journal of Personality and Social Psychology, 46: 1349-1364. 
List, J. A. (2007). On the interpretation of giving in dictator games. The Journal of Political Economy 115(3), 482-493.

Lu, Y., Westfall, P. H., Han, G., and Bui, M. M. (2016). Bayesian hypothesis testing for selected regression coefficients. Communications in Statistics - Theory and Methods, 45, 7011-7026.

Maclagan, P. (1990). Moral behavior in organizations: The contribution of management education and development. British Journal of Management, 1: 17-26.

Mayer, J. D. and Salovey, P. (1997). What is emotional intelligence? In P. Salovey \& D. J. Sluyter (Eds.), Emotional development and emotional intelligence (3-31). New York, Basic Books.

Mulki, J., Jaramillo, J., and Locker, W. (2009). Critical role of leadership on ethical climate and salesperson behaviors. Journal of Business Ethics, 86(2): 125-141.

$\mathrm{Ng}, \mathrm{K}$, Wang, C. and Zalaquett, C. (2008). A confirmatory factor analysis of the Wong and Law Emotional Intelligence Scale in a sample of international college students. International Journal of Advanced Counseling 30: 131-144.

Rest, J. (1993). Guide to using the DIT, version 1.3. Center for the Study of Ethical Development, University of Minnesota.

Rest, J. and Narváez, D. (1998). Guide for DIT-2. Minneapolis, MN: Center for the Study of Ethical Development, University of Minnesota.

Ross, W. T. and Robertson, D. C. (2003). A Typology of situational factors: Impact on salesperson decision-making about ethical issues, Journal of Business Ethics, 46(3), 213-234.

Salovey, P. \& Mayer, J. D. (1990). Emotional intelligence. Imagination, Cognition, and Personality, 9: 185-211.

Snyder, M. (1979). Self-monitoring processes. Advances in Experimental Social Psychology, 12: 85-128.

Van Rooy, D. L. and Viswesvaran, C. (2004). Emotional intelligence: A meta-analytic investigation of predictive validity and nomological net. Journal of Vocational Behavior 65(2004) 71-95.

Weaver, G. and Trevino, L. (1999). Compliance and values oriented ethics programs: Influences on employee's attitudes and behavior. Business Ethics Quarterly, 9(2): 315-335.

Whitman, D. S., VanRooy, D. L., Viswesvaran, C., and Kraus, E. (2009). Testing the secondorder factor structure and measurement equivalence of the Wong and Law Emotional Intelligence Scale across gender and ethnicity. Educational and Psychological Measurement, 69(6): 1059-1074.

Wong, C. S., and Law, K. S. (2002). The effects of leader and follower emotional intelligence on performance and attitude: An exploratory study. Leadership Quarterly, 13, 243-274.

Wright, M.(1995). Can moral judgment and ethical behavior be learned? Management Decision, 10(17): 17-29. 